give more useful numbers. For birds and mammals, this line of analysis implies extinction of half the species within roughly 200-300 years, and for palms it suggests $50-100$ years.

We re-emphasize that the data in the Red Lists have been compiled opportunistically rather than systematically, so that these projected rates of extinction probably owe more to the vagaries of sampling effort and data entry than to real changes in the status of species. However, we believe that the approach does have potential merit in suggesting a new line of investigation of the dynamics of species extinction. The consequent estimates of extinction rates for the better-known taxa

1. Wilson, E. O. The Diversity of Life (Belknap, Cambridge Massachusetts, 1992)

2. Diamond, J. M. Phil. Trans. R. Soc. B325, 469 (1989).

3. Wilson, E. O. In Putting Biodiversity on the Map Priority Areas for Bird Conservation vi (International Council for Bird Preservation Cambridge, 1992).

4. World Conservation Monitoring Centre Global Biodiversity: Status of the Earth's Living Resources (Chapman \& Hall, London, 1992).

5. International Union for the Conservation of Nature Red List of Threatened Animals compiled by WCMC (IUCN. Gland, 1986). are of the same order of magnitude as those derived from totally unrelated species-area relations, and they suggest that the time available for research into other plant and animal groups may be severely limited.

Fraser D. M. Smith

Robert M. May

Department of Zoology,

University of Oxford,

South Parks Road, Oxford OX1 3PS, UK

Robin Pellew

Timothy H. Johnson

Kerry S. Walter

World Conservation Monitoring Centre,

219 Huntingdon Road,

Cambridge CB3ODL, UK

6. International Union for the Conservation of Nature Red List of Threatened Animals compiled by WCMC (IUCN, Gland, 1988)

7. International Union for the Conservation of Nature Red List of Threatened Animals compiled by WCMC (IUCN, Gland, 1990)

8. Gaston, K. J. \& May, R. M. Nature 356, 281-282 (1992)

9. Shirt, D. B. British Red Data Book No. 2: Insects Joint Nature Conservation Committee (1987)

10. May, R. M. Scient. Am. 268, 146-149 (March 1993).

\title{
Triplex model of chromosome ends
}

SIR - Sundquist and $\mathrm{Klug}^{1}$ demonstrated that synthetic models of Tetrahymena chromosome telomeric terminus, consisting of the DNA duplex and the singlestranded 'tail' $\left(\mathrm{T}_{2} \mathrm{G}_{4}\right)_{2}$, stably dimerize in monovalent salts owing to quadruplex formation by the two tails. We have found that in physiological buffer containing about $100 \mathrm{mM} \mathrm{Na}^{+}$and $10 \mathrm{mM} \mathrm{Mg}^{2+}$ the

also virtually fully protected. Some protection is visible also for the terminal block of the tail.

At $10{ }^{\circ} \mathrm{C}$ and in the presence of $\mathrm{Mg}^{2+}$, the molecules move in the gel as a single band with mobility corresponding to the mobility of monomers (data not shown), although we observed formation of dimers described by Sundquist and Klug ${ }^{1}$ in experiments without magnesium after long incubation.

3' - CAGTTCGAACCAACCCCAACCCCAACCCC 5' - GTCAAGCTTGCTTGGGGTTGGGGTTGGGG T

3. - GGGGTTGGGG-T

FIG. 1 Synthetic Tetrahymena chromosome terminus forming the proposed foldback triplex structure.

same model of chromosome ends forms an intramolecular pyrimidine-purine-purine triplex: the single-stranded G-rich overhang folds back, lying into the major groove of the terminal duplex forming the CGG base triads (Fig. 1). This new structure of telomeres provides a possible explanation for the stability of the ends of chromosomes in vivo against digestion and recombination.

Figure 2 presents our data on probing of the structure with dimethyl sulphate (DMS). Without $\mathrm{Mg}^{2+}$, we observe homogeneous modification of guanines along the whole G-rich strand (lanes 1-3). In the presence of $\mathrm{Mg}^{2+}$, a completely different pattern of modification is observed: one can see the clear-cut protection of guanines in two blocks within the duplex adjacent to single-stranded tails (lanes 4-6). Moreover, at least one of two guanine blocks belonging to the tail is
For the incubation times we used in our experiments in Fig. 2, dimers never form (our unpublished observations and ref. 1). This means that the clear-cut protection effect against methylation in Fig. 2 results from an internal structural rearrangement in the molecule. We therefore assume that in the presence of $\mathrm{Mg}^{2+}$ ions the singlestranded tail folds back, forming a pyrimidine-purine-purine triplex consisting of the CGG base triads (Fig. 1).

The model in Fig. 1 explains all major features of the results in Fig. 2 and is fully consistent with the available data on the CGG type of pyrimidine-purine-purine triplexes $^{2-5}$. Formation of the pyrimidinepurine-purine triplex leads to the complete protection of guanines in the duplex against modification by $\mathrm{DMS}^{4,5}$ and, as we have recently shown, formation of the CGG base triad leads to significant protection of guanines in the third strand ${ }^{4}$.

Our foldback triplex model provides a natural explanation for the stabilizing effect of chromosome ends. Occupation of the major groove and concomitant

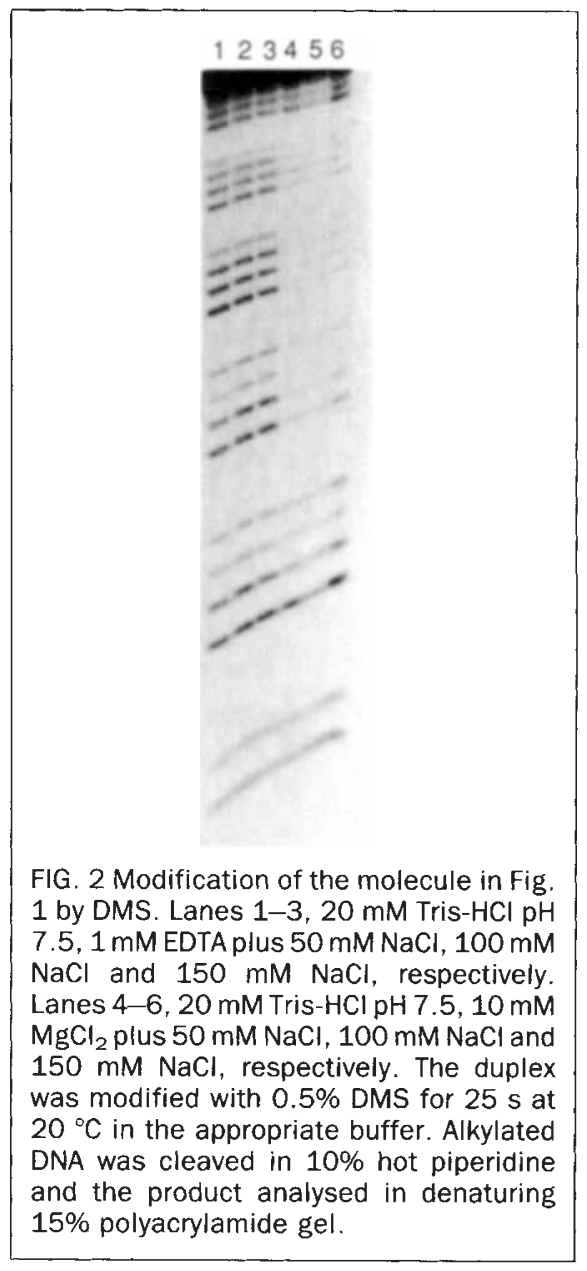

deformation of the minor groove due to triplex formation at the very end of chromosomal DNA should dramatically decrease vulnerability of the ends of chromosomes as targets for enzymatic cleavage. One can also assume that the foldback triplex structure would protect the telomeric ends against digestion by both $5^{\prime}-3^{\prime}$ and $3^{\prime}-5^{\prime}$ exonucleases. It is also quite reasonable to assume that the structure in Fig. 1 would prevent the telomeric ends from participating in recombination events.

Alexel G. Veselkov

Vladislav A. Malkov

Maxim D. Frank-Kamenetskli

Institute of Molecular Genetics,

Russian Academy of Science,

Moscow 123182, Russia

Vladimir N. Dobrynin

Shemyakin Institute of Bioorganic Chemistry,

Russian Academy of Sciences,

Moscow, Russia

1. Sundquist. W. I. \& Klug, A. Nature 342, 825-829 (1989).

. Kohwi, Y. \& Kohwi-Shigematsu, T. Proc. natn. Acad. Sci U.S.A. 85, 3781-3785 (1988)

3. Malkov V. A., Soyfer, V. N. \& Frank-Kamenetskii, M. D. Nucleic Acids Res. 20, 4889 -4895 (1992).

4. Maikov, V. A. etal. Nucleic Acids Res. 21, 105-111 (1993).

5. Malkov, V. A., Voloshin, O. N., Soyfer, V. N. \& Frank-Kamenetskii, M. D. Nucleic Acids. Res. 21 585-591 (1993) 